\title{
"Hospital donante" una estrategia educativa: experiencia de un centro latinoamericano de trasplantes
}

\author{
Karen Torres ${ }^{1}$, Ana M. Aristizábal ${ }^{2}$, Gabriel J. Echeverri ${ }^{3,8}$, Santiago Cuenca ${ }^{1}$, Mariana Roldán ${ }^{4}$, \\ Viviana Escobar ${ }^{5}$, Cristina González ${ }^{6}$, Patricia Echeverry ${ }^{7}$, Yenni Ramírez ${ }^{7}$, Víctor García ${ }^{2}$, \\ Catalina Gómez ${ }^{2}$, Luis A. CAicedo ${ }^{3}$
}

Palabra clave: hospitales; trasplante; trasplante de órganos; selección de donante; obtención de tejidos y órganos.

\section{Resumen}

Introducción. Los trasplantes de órganos son el único tratamiento para pacientes con enfermedades crónicas avanzadas. Sin embargo, existe una disparidad entre el número de pacientes en lista de espera y el número de

1 Médica, coordinadora operativa de trasplantes, Fundación Valle del Lili, Cali, Colombia

2 Médica, investigadora, Centro para la Investigación en Cirugía Avanzada y Trasplantes, Universidad Icesi; Centro de Investigaciones Clínicas, Fundación Valle del Lili, Cali, Colombia

3 Médico, especialista en Cirugía de Trasplante de Órganos Abdominales, Fundación Valle del Lili, Cali, Colombia

4 Comunicadora social, Fundación Valle del Lili, Cali, Colombia

5 Psicóloga clínica, Unidad de Trasplantes, Fundación Valle del Lili, Cali, Colombia

6 Enfermera coordinadora de trasplantes, Fundación Valle del Lili, Cali, Colombia

7 Coordinadora administrativa de trasplantes, Fundación Valle del Lili, Cali, Colombia

8 Director, Centro para la Investigación sobre Cirugía Avanzadas y Trasplantes (CICAT), Universidad Icesi, Cali, Colombia

Fecha de recibido: 22 de enero de 2017

Fecha de aprobación: 26 de mayo de 2017

Citar como: Torres K, Aristizábal AM, Echeverri GJ, Cuenca S, Roldán M, Escobar V, et al. "Hospital donante" una estrategia educativa: experiencia de un centro latinoamericano de trasplantes. Rev Colomb Cir. 2017;32:205-13. donantes. Una forma de solucionar esto es mediante la educación y la sensibilización de la comunidad.

El objetivo de este estudio fue evaluar el impacto que tuvo una campaña de promoción de la donación en un centro de trasplantes colombiano.

Métodos. Se hizo una encuesta en la institución sobre el conocimiento previo y las creencias sobre la donación. Posteriormente, se diseñó una estrategia para informar, educar y promover la donación y, finalmente, se llevó a cabo un estudio transversal para evaluar el impacto de la campaña.

Resultados: hubo un incremento del $56 \%$ de potenciales donantes, así como de la obtención de órganos y tejidos: $100 \%$ de órganos sólidos, $200 \%$ de tejido ocular y $850 \%$ de tejido óseo.

Discusión. El conocer la actitud hacia la donación permite determinar factores que influyen en la toma de decisiones y así elaborar campañas que generen un aumento en los índices de donación. Según nuestro estudio, el generar una cultura a favor de la donación tiene un alto impacto en los índices de donación, y una posible causa de los bajos índices podría corresponder a la falta de información que tienen las personas, incluyendo los trabajadores de la salud. Este trabajo puede ser extrapolado a nivel nacional para conocer las causas de las negativas a donar y adoptar politicas que aumenten los índices de donación. 


\section{Introducción}

Existe un porcentaje considerable de la población colombiana que no cuenta con un acceso oportuno o adecuado al sistema de salud, especialmente a los programas de promoción y prevención. La mayoría de los pacientes que sufren de enfermedades crónicas, son remitidos a un servicio especializado cuando su enfermedad se encuentra avanzada o en un estadio en el cual el manejo debe ser agresivo y radical.

La enfermedad renal crónica se ha convertido en un problema de salud pública para la población mundial, pues afecta a 1 de cada 10 adultos en el mundo ${ }^{1}$. En Colombia, en el año 2013, por cada 100.000 afiliados al Sistema General de Seguridad Social en Salud, 65,88 tenían diagnóstico de enfermedad renal crónica en estadio $5^{1}$.

Los trasplantes de órganos pueden constituir el único tratamiento curativo para las enfermedades crónicas avanzadas. No obstante, el número de pacientes en lista de espera para trasplante aumenta día a día de manera exponencial $\mathrm{y}$, por el contrario, el número de donantes disminuye.

Según cifras del Instituto Nacional de Salud de Colombia, en el 2015 hubo un total de 405 donantes reales de órganos, para una tasa nacional de 8,4 donantes por millón de habitantes, la cual fue, en su mayoría, a expensas de donantes cadavéricos ${ }^{2}$. La lista de espera del Instituto Nacional de Salud a 31 de diciembre del 2015, reportaba 2.029 pacientes que necesitaban órganos sólidos para trasplante y 1.204 pacientes recibieron un trasplante en el país en ese año ${ }^{2}$.

En el mundo existen diferentes programas de promoción de la donación de órganos y tejidos que demuestran ser una herramienta útil en el aumento de los índices de donación ${ }^{3-8}$. Igualmente, estos programas generan una expansión exponencial de los conocimientos, puesto que todo aquel que cuenta con la información acertada puede ser difusor del tema ${ }^{9,10}$.

El modelo español de coordinación y trasplantes demuestra la importancia de la educación y de la sensibilización de la población para mejorar la actitud y fomentar el diálogo socio-familiar sobre este tema, lo que aumenta el número de donantes y disminuye el tiempo de espera para los trasplantes ${ }^{11}$. Asimismo, se han creado campañas de donación de órganos en las mezquitas, para superar las barreras y la desinformación que tiene la población que profesa la religión musulmana ${ }^{12}$.

El objetivo de este estudio fue evaluar el impacto que tuvo una campaña de promoción de la donación de órganos y tejidos realizada en la Clínica Fundación Valle del Lili, centro hospitalario de cuarto nivel ubicado en Cali y con amplia experiencia en trasplantes ${ }^{13-25}$. Es una institución en donde hace más de 20 años está en marcha el programa de trasplantes, la única institución prestadora de salud (IPS) en Colombia donde se hace trasplante simultáneo de riñón y páncreas y, en la actualidad, se practican trasplantes de hígado, páncreas, islotes pancreáticos, riñones, intestino, pulmón y corazón.

\section{Métodos}

Se hizo una encuesta sobre la donación de órganos y tejidos, de manera aleatoria, a 288 trabajadores del área de la salud o colaboradores administrativos de la Clínica Fundación Valle del Lili. El número de encuestas se determinó con base en el criterio de saturación, el cual se refiere al momento en que el material cualitativo deja de aportar datos nuevos y hace más dispendioso el análisis de los datos cualitativos ${ }^{26}$.

En dicho formato, se emplearon técnicas cualitativas para recolectar la información; se determinó utilizar este método, debido a que se buscaba profundizar en la caracterización de los conocimientos y creencias en salud, en torno al tema de la donación de órganos y tejidos ${ }^{26}$. La entrevista contó con 15 preguntas sobre el conocimiento previo, mitos y creencias sobre el tema (anexo 1).

Entre los criterios de inclusión, se tuvieron en cuenta trabajadores de sexo femenino y masculino de la Clínica Fundación Valle del Lili entre los 20 y los 40 años de edad. Aunque no se trató de un muestreo representativo de la población, se trató de incluir informantes que reflejaran distintas características demográficas (estrato social, nivel educativo y ocupación, entre otras).

Posteriormente, se diseñó una estrategia que relacionó tres propósitos: informar, educar y comunicar para promover la donación de órganos y tejidos. El objetivo final de esta campaña era derribar las barreras identificadas al analizar las respuestas obtenidas en la encuesta, crear 
una cultura a favor de la donación, y aumentar el índice de donación de órganos y tejidos en la Fundación.

Se crearon un logotipo y un eslogan como imágenes representativas de la campaña. Utilizando estos diseños, se imprimieron 2.000 afiches, 1.000 folletos (anexo 2), 500 globos, 500 porta-documentos, 2.500 adhesivos para dulces, 1.600 carnés del Instituto Nacional de Salud, 1 pendón y 4 rompe-tráficos, además de 24 bonsái con tarjetas de agradecimiento para ser entregados a las familias de los donantes.

Aparte de todo el material diseñado, se dictaron 32 charlas magistrales a estudiantes de Medicina de la Universidad Icesi, médicos generales y especialistas de las diferentes áreas de la clínica (especialmente urgencias y unidades de cuidado intensivo), personal de enfermería y fisioterapia, colaboradores de servicios generales y personal del área administrativa. Por medio de las charlas, se buscaba explicar la importancia del proceso de donación y aclarar dudas de los mitos y realidades de la donación.

Asimismo, se hizo promoción de la campaña en 11 eventos educativos realizados en la Fundación, brindando información y dictando conferencias sobre la donación de órganos y tejidos en cada uno de ellos.

Finalmente, se hizo un estudio de corte transversal para evaluar el impacto que tuvo esta campaña educativa en un periodo de 12 meses. El impacto se analizó midiendo la variación en el número de alertas de potenciales donantes y el número de trasplantes realizados durante el 2014 y el 2015 en la Fundación.

\section{Análisis estadístico}

Las variables cualitativas categóricas se analizaron mediante proporciones y para cada propósito de interés. Las variables cuantitativas continuas se analizaron mediante medidas de tendencia central con promedio y desviación estándar (DE) y mediana con rango intercuartílico (RIQ), dependiendo de la distribución de los datos según la prueba de Shapiro-Wilk. Se hicieron comparaciones entre las alertas de potenciales donantes y la obtención de órganos y tejidos durante cada mes de los años evaluados.

\section{Resultados}

$\mathrm{Al}$ analizar las respuestas de las 288 encuestas, se encontró que el $97 \%$ de los encuestados sabía qué es la donación de órganos y tejidos, el $93 \%$ serían donantes y el $69 \%$ reconoció haber hablado con su familia del tema.

E1 $2 \%$ de los encuestados no estaba a favor de la donación. Entre los argumentos para negarse a la donación, se identificaron: aspectos religiosos (23\%), considerar que 'es un negocio' $(22 \%)$, considerar que 'no es la cura para el receptor' $(22 \%)$, creer que 'lo secuestrarían para robarle los órganos y tejidos' (11\%), no tener suficiente información $(11 \%)$ y no desear que le retiren ningún órgano o tejido (11\%).

Al interrogar si donaría en vida algún órgano a un familiar, el $96 \%$ respondió que lo haría, mientras que el $4 \%$ no donaría, principalmente por temor a la cirugía y sus complicaciones. El $91 \%$ de los encuestados, si tuvieran la oportunidad, donarían los órganos y tejidos de algún familiar si falleciera.

Por otro lado, se encontró que el $73 \%$ cree que el tráfico de órganos existe en Colombia y el $17 \%$ asume que hay algún beneficio económico por la donación.

El $33 \%$ de los encuestados no sabe en dónde encontrar información sobre el tema, lo cual concuerda con el $44 \%$ que no sabe cómo convertirse en donante de órganos y tejidos. El 90 \% solicitó mayor información sobre el tema.

En cuanto al impacto que tuvo la campaña de educación y promoción, se evidenció un incremento del $56 \%$ en las alertas de potenciales donantes, especialmente, en el último semestre del año 2015 (figura 1). La consecución de órganos tuvo una variación del $100 \%$, en el 2014 se presentaron 15 rescates y 30 en el 2015 (figura 2).

Con respecto a la obtención de tejidos, se evidenció un incremento considerable. Hubo una variación del 200 $\%$ entre los años 2014 y 2015 en la consecución de tejido ocular, con 45 rescates durante el 2015 y 15 durante el 2014 (figura 3). Con relación a la obtención de tejido óseo, hubo un incremento del $850 \%$, esto debido a que en el año 2014 se registraron dos rescates, mientras que a lo largo del 2015 fueron 19 en total (figura 4). 


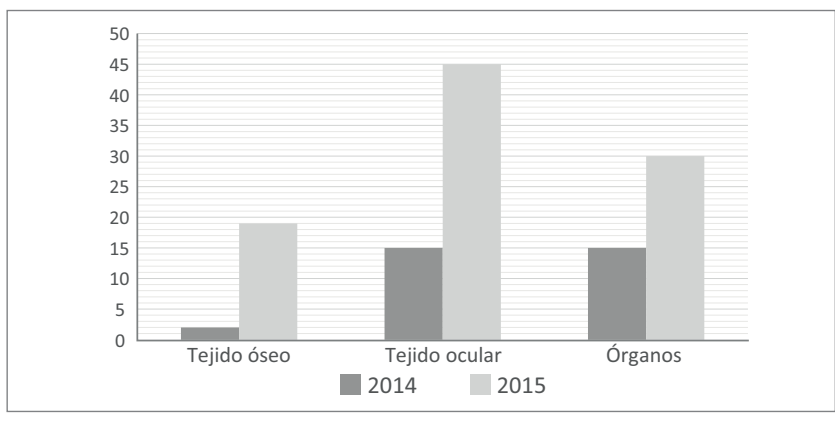

Figura 1. Variación de las alertas de potenciales donaciones, Unidad de Trasplantes, Fundación Valle del Lili, 2014 y 2015

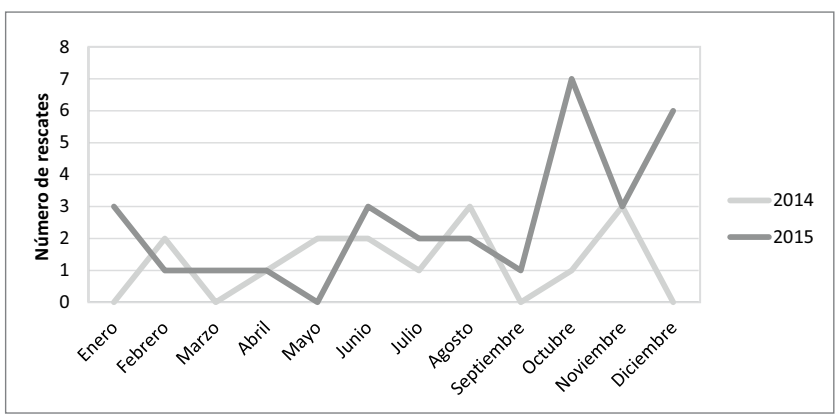

Figura 2. Variación de la consecución de órganos, Unidad de Trasplantes, Fundación Valle del Lili, 2014 y 2015

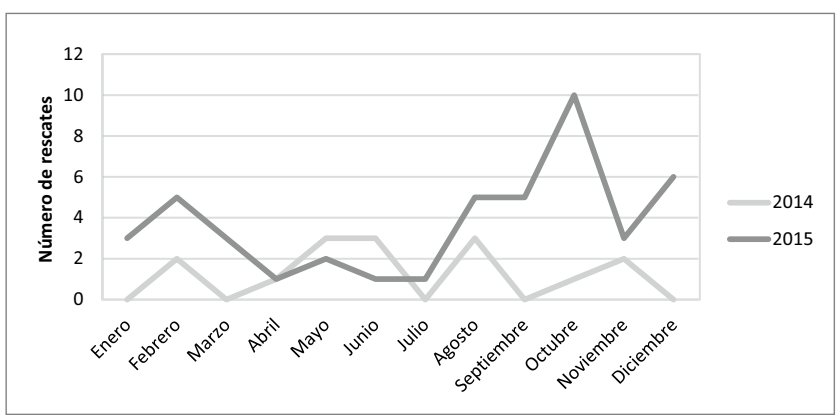

Figura 3. Variación de la obtención de tejido ocular, Unidad de Trasplantes, Fundación Valle del Lili, 2014 y 2015

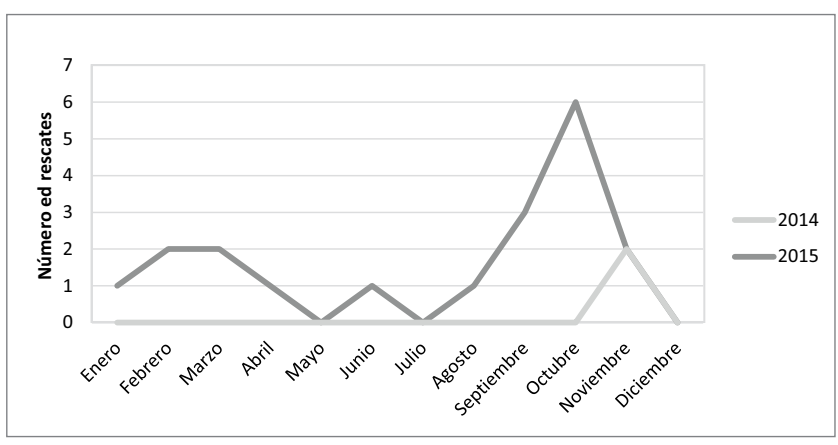

Figura 4. Variación de la obtención de tejido óseo, Unidad de Trasplantes, Fundación Valle del Lili, 2014 y 2015

\section{Discusión}

Los trasplantes de órganos se han consolidado como el tratamiento más eficiente y que mejor calidad de vida brinda a los pacientes con la función de un órgano en estadio terminal ${ }^{27-29}$. Sin embargo, hay una escasez de órganos disponibles, lo que genera largas listas de espera $\mathrm{y}$ hace que solo un porcentaje pequeño de los pacientes pueda recibir un trasplante ${ }^{7}$.

Esta carencia de órganos para trasplante ha venido siendo combatida con la donación de órganos procedentes de donantes vivos y con el esfuerzo, en algunos países, por aumentar las tasas de donación de órganos procedentes de donantes cadavéricos ${ }^{30}$. El conocer la actitud hacia la donación de órganos permite determinar los factores que influyen en la toma de decisiones y, así, elaborar campañas adecuadamente diseñadas y costo-efectivas que generen un aumento en los índices de donación ${ }^{7}$.

Cabe resaltar que las campañas de educación dirigidas a la comunidad y, sobre todo a los trabajadores del área de la salud, tienen un gran impacto ${ }^{31-33}$. Infortunadamente, en la mayoría de las facultades del área de la salud, el currículo no incluye temas como la donación de órganos y tejidos, y no todos los profesionales de la salud saben del tema. Además, en la sociedad se generan mitos y creencias falsas a partir de vivencias personales, que crean una imagen distorsionada del proceso de donación de órganos y tejidos.

Debido a lo anterior, la campaña "Hospital donante" que adelantamos en nuestra institución, se centró en derribar las barreras del proceso de la donación por medio de información al respecto. Consideramos que el personal de la Fundación debe estar muy bien capacitada sobre la donación, ya que el programa de trasplantes lleva en marcha más de 20 años y ha tenido excelentes resultados ${ }^{21,34}$.

Entre las limitaciones de nuestro estudio, reconocemos que la encuesta utilizada no se encuentra validada; fue elaborada por el equipo de comunicación social y psicología de nuestra unidad, con el fin de averiguar el conocimiento y las creencias que tenían los trabajadores de la institución. La encuesta se llevó a cabo de manera aleatoria en un porcentaje no representativo de la Fundación, debido a que era un material cualitativo y el criterio de exclusión determina que aplicarla a un número mayor de personas no aporta nuevos datos. 
Según los resultados obtenidos, se podría sugerir que, probablemente, una de las causas del bajo índice de donación que tiene la región, corresponde a la falta de información o a las falsas creencias que tienen las personas, incluyendo aquellos que trabajan en el área de la salud.

Nuestro centro médico es una de las dos IPS de la región en donde se hacen trasplantes. En teoría, el personal debería tener un adecuado conocimiento del tema; no obstante, encontramos que hay mucha desinformación y falsas creencias. Por lo anterior, si esto sucede en una IPS donde se practican trasplantes, es probable que exista este mismo problema, o incluso peor, en el resto de la región.

Por medio de nuestra campaña, se demostró que el generar una cultura a favor de la donación de órganos y tejidos, dando la información adecuada y aclarando las dudas sobre el tema, tiene un alto impacto en el número de alertas de potenciales donantes, los cuales, en un importante porcentaje, terminan convirtiéndose en donantes multiorgánicos efectivos y mejorando los índices de donación.

Es importante reconocer que la formación y la concientización de la población deben ser constantes. Asimismo, deben abarcar a toda la población, desde el inicio de la formación educativa, siendo buenas opciones, los colegios o las universidades, con el fin de generar una cultura a favor de la donación desde temprana edad.

En conclusión, este modelo de campaña educativa demostró tener excelentes resultados en una IPS del Valle del Cauca en donde se practican trasplantes, aumentando las tasas de donación y de donantes efectivos. Este trabajo podría ser extrapolado a nivel regional y nacional, con el fin de conocer las causas de las negativas de la donación $\mathrm{y}$, posteriormente, adoptar políticas públicas que permitan el aumento de los índices de donación.

\title{
“Donor hospital” an educational strategy: experience at a Latin American transplant center
}

\begin{abstract}
Introduction: Organ transplant is the only effective treatment for patients with advanced chronic disease. Although there is a gap between the number of patients in the transplant waiting lists and the number of donors, one way to diminish the waiting lists is by educating and creating awareness in the community. The objective of this study was to evaluate the impact of an institutional campaign that encourages organ donation at a Colombian transplant center.

Methodology: An institutional survey was carried out in order to assess the prior knowledge, myths and beliefs around organ and tissue donation. Subsequently, a plan was designed to inform, educate and promote organ donation within the institution. Finally, a cross-sectional study was carried out to evaluate the impact of the campaign during the years of 2014-2015.

Results: There was an increase of $56 \%$ in the number of potential donors as well of as of organ and tissue procurements: $100 \%$ of solid organs, $200 \%$ ocular tissue and $850 \%$ bone tissue.

Discussion: Knowing the attitude towards organ and tissue donation allows to determine factors that influence decision making and to develop campaigns that generate an increase in donation rates. According to our study, generating a culture for organ donation has a high impact on donation rates, and a possible cause of the low rates could correspond to the lack of information that people have, including health workers. This work could be extrapolated nationally in order to recognize the causes of donation denials and to adopt policies that increase donation rates.
\end{abstract}

Key words: Hospitals; transplantation; organ transplantation; donor selection; tissue and organ procurement. 


\section{Referencias}

1. Ministerio de Salud y Protección Social. Análisis de situación de salud. Bogotá: Imprenta Nacional de Colombia; 2014. p. 235.

2. Ospina ML, Beltrán M, Segura A. Informe anual, Red de Donación y Trasplantes, Colombia, 2015. Bogotá: Instituto Nacional de Salud; 2016. Fecha de consulta: 23 de enero de 2017. Disponible en: http://www.consultorsalud.com/sites/consultorsalud/ files/informe_ejecutivo_red_donacion_y_trasplantes_2015.pdf.

3. Yilmaz TU. Importance of education in organ donation. Exp Clin Transplant 2011;9:370-5.

4. Cantarovich F, Heguilén R, Filho MA, Duro-García V, Fitzgerald R, Mayrhofer-Reinhartshuber D, et al. An international opinion poll of well-educated people regarding awareness and feelings about organ donation for transplantation. Transpl Int. 2007;20:512-8.

5. Cantarovich M, Birk P, Ekbeg H, Delmonico F, Schoenberg $\mathrm{R}$, García $\mathrm{C}$, et al. First global forum on education on organ donation and transplantation for schools. Pediatr Transplant. $2013 ; 17: 12-8$.

6. Li AH-T, Rosenblum AM, Nevis IF, Garg AX. Adolescent classroom education on knowledge and attitudes about deceased organ donation: A systematic review. Pediatr Transplant. 2013;17:119-28.

7. Ríos A, López-Navas A, Ayala-García MA, Sebastián MJ, Abdo-Cuza A, Alán J, et al. Spanish-Latin American multicenter study of attitudes toward organ donation among personnel from hospital healthcare centers. Cirugía Española. 2014;92:393-403.

8. Ríos-Zambudio A, López-Navas A, Ayala-García M, Sebastián MJ, Abdo-Cuza A, Alán J, et al. Level of awareness of personnel in hospital services related to the donation process: A Spanish and Latin American multicenter study. J Heart Lung Transplant. 2012;31:850-7.

9. Walker W, Sque M. Balancing hope and despair at the end of life: The contribution of organ and tissue donation. J Crit Care. 2016;32:73-8.

10. Ríos A, Febrero B, López-Navas A, Martínez-Alarcón L, Sánchez J, Guzmán D, et al. Secondary school teachers' assessment of the introduction of an educational program about organ donation and transplantation. Transplant Proc. 2011;43:58-60.

11. Ríos A, Ramírez P, Martínez L, Montoya MJ, Lucas D, Alcaraz J, et al. Are personnel in transplant hospitals in favor of cadaveric organ donation? Multivariate attitudinal study in a hospital with a solid organ transplant program. Clin Transplant. 2006;20:743-54.

12. Rady MY, Verheijde JL. Campaigning for organ donation at mosques. HEC Forum. 2016;28:193-204.

13. Holguín A, Rodríguez-Takeuchi S, Ospina L, Acosta D, Botero $\mathrm{V}$, Thomas L, et al. Biliary strictures complicating liver transplantation in pediatric patients: Experience in a South American transplant center. Clin Transplant. 2017;31:e12877. doi: 10.1111/ ctr.12877.
14. Caicedo L, Gómez JC, Serrano O, Manzi E, Posada JG, Mesa L, et al. Derivación exocrina al duodeno en trasplante simultáneo de riñón y páncreas, experiencia en la Fundación Valle del Lili, Cali, Colombia. Rev Colomb Cir. 2016;31:178-84.

15. Millán M, Delgado A, Caicedo LA, Arrunátegui AM, Meneses CA, Villegas JI, et al. Liver angiosarcoma: Rare tumour associated with a poor prognosis, literature review and case report. Int J Surg Case Rep. 2016;28:165-8.

16. Millán M, Caicedo LA, Villegas JI, Serrano O, Caicedo L, Duque $\mathrm{M}$, et al. Case report of cadaveric kidney transplantation with renal-portal venous drainage: A feasible way for a venous drainage in a complex generalized thrombosed vessels setting. Int J Surg Case Rep. 2016;28:192-5.

17. Serrano ÓJ, Villegas JI, Echeverri GJ, Posada JG, Mesa L, Schweineberg J, et al . Trasplante simultáneo de riñón y páncreas en pacientes con diabetes mellitus de tipo 1, Clínica Fundación Valle del Lili, Cali, 2001-2013. Rev Colomb Cir. 2014:29:32-41.

18. Sabogal A, Casas LA, Arango LG, Feriz K, Guzmán G, Gutiérrez Ó, et al. Presente y futuro del trasplante de islotes pancreáticos, un tratamiento innovador para la diabetes tipo 1. Rev Colomb Endocrinol Diabetes Metab. 2015;2:20-30.

19. Echeverri GJ, Sabogal AN, Caicedo LA, Casas LA, Mesa L, Schweineberg J, et al. Primer trasplante de islotes realizado en Colombia, experiencia Fundación Valle del Lili. Rev Colomb Endocrinol Diabetes Metab. 2015;2:33-9.

20. Caicedo LA, Gómez JC, Duque MF, Serrano ÓJ, Manzi E, Arrunátegu AM, et al. Trasplante renal con HLA idéntico de donante vivo y cadavérico: experiencia de la Fundación Valle del Lili, Cali, Colombia. Rev Colomb Cir. 2016;31:170-7.

21. Caicedo LA, Vernaza C, Valderrama ML, Manzi E, Echeverri GJ, Jiménez DF, et al. The experience of the Fundacion Valle del Lili in Cali, Colombia, with combined liver and kidney transplantation. Rev Col Gastroenterol. 2016;31:96-101.

22. Caicedo LA, Villegas JI, Serrano O, Millán M, Sepúlveda M, Jiménez D, et al. En-bloc transplant of the liver, kidney and pancreas: Experience from a Latin American transplant center. Am J Case Rep. 2017;18:114-8.

23. Aristizábal AM, Caicedo LA, Martínez JM, Moreno M, Echeverri GJ. Xenotrasplantes, una realidad cercana en la práctica clínica: revisión de la literatura. Cir Esp. 2017;95:62-72.

24. Caicedo LA, Buitrago D, Thomas LS, Villegas JI, Duque M, Serrano O, et al. Unresectable metastases from colon adenocarcinoma: A new indication for liver transplantation. Case Rep Gastroenterol. 2016;10:808-13.

25. Caicedo L, Sabogal A, Serrano O, Villegas J, Botero V, Agudelo $\mathrm{M}$, et al. Hepatoblastoma: Transplant versus resection experience in a Latin American transplant center. Transplant Direct. 2017;3:e165.

26. Roldán M. Diseño de una estrategia de información, educación y comunicación (IEC) para promover la donación de órganos 
y tejidos en la Fundación Valle del Lili en Cali (tesis). Cali: Pontificia Universidad Javeriana; 2015.

27. Mejía D, Ramírez P, Ríos A, Munitiz V, Hernández Q, Bueno F, et al. Recurrence of alcoholism and quality of life in patients with alcoholic cirrhosis following liver transplantation. Transplant Proc. 1999;31:2472-4.

28. De Baere C, Delva D, Kloeck A, Remans K, Vanrenterghem Y, Verleden G, et al. Return to work and social participation: Does type of organ transplantation matter? Transplantation. 2010;89:1009-15.

29. López-Navas A, RÍos A, Riquelme A, Martínez-Alarcón L, Pons JA, Miras M, et al. Importance of introduction of a psychological care unit in a liver transplantation unit. Transplant Proc. 2010;42:302-5.

30. Council of Europe. International figures on donation and transplantation 2009. Newsletter Transplant. 2010;15:1-70.

31. Tumin M, Tafran K, Tang LY, Chong MC, Mohd Jaafar NI, Mohd Satar N, et al. Factors associated with medical and nursing students' willingness to donate organs. Medicine (Baltimore). 2016;95:e3178.
32. Dalal AR. Philosophy of organ donation: Review of ethical facets. World J Transplant. 2015;5:44-51.

33. Vertanous T, Czer LSC, de Robertis M, Kiankhooy A, Kobashigawa J, Esmailian F, et al. Leading efforts to increase organ donation through professionalization of organ procurement organizations and establishment of organ and tissue donor registries. Transplant Proc. 2016;48:10-4.

34. Serrano Ó, Villegas J, Echeverri G, Posada J, Mesa L, Schweineberg J, et al. Simultaneous kidney and pancreas transplantation in patients with type 1 diabetes mellitus at Clínica Fundación Valle del Lili, Cali, Colombia. Rev Colomb Cir. 2014;29:32-41.

\section{Correspondencia:}

Gabriel J. Echeverri, MD

Correo electrónico: gjecheverri@hotmail.com Cali, Colombia 
• जाए厂 VALLE DEL LILI

Excelencia en Salud al servicio de la comunidad
Edad:

Sexo:

Ocupación:

Escolaridad:

\section{ENCUESTA INTERNA UNIDAD DE TRASPLANTES}

1. ¿Sabe qué es la donación de órganos y tejidos?

2. ¿Alguna vez le han hablado sobre la donación de órganos y tejidos?

3. ¿Está de acuerdo con la donación de órganos y tejidos?

4. ¿En su familia han hablado sobre donar órganos y tejidos?

5. ¿Donaría sus órganos y tejidos?

Si su respuesta en NO, explique la razón por la que no está de acuerdo con la donación de órganos y tejidos, según el listado que se relaciona a continuación.

- Aspectos religiosos

- Me secuestran para robarme los órganos y tejidos.

- Piensa que es negocio.

- No es una cura real para el paciente que lo recibe.

- Otra

6. ¿Donaría en vida un órgano para un familiar?

7. Del siguiente listado, ¿usted qué donaría?

- Hígado

- Riñones

- Corazón

- Pulmón

- Laringe y tráquea

- Córneas

- Hueso

- Piel

- Válvulas cardiacas

8. Si de la lista anterior marcó alguno con NO, por favor, explique porqué

9. ¿Donaría los órganos o tejidos de algún familiar después de fallecer, si le hablan de esta posibilidad?

10. ¿Cree que existe el tráfico de órganos y tejidos?

11. ¿Cree que existe algún benefício económico al donar?

12. ¿Sabe dónde encontrar información sobre la donación de órganos y tejidos?

13. ¿Cómo se enteró sobre la donación de órganos y tejidos?

- Internet

- Publicidad (afiches, volantes)

- Charlas

- Iniciativa propia

- Otras

14. ¿Sabe cómo volverse donante de órganos y tejidos?

15. ¿Le gustaría recibir información sobre la donación de órganos y tejidos?

ANEXo 1. Encuesta sobre la donación de órganos y tejidos "Hospital donante" 

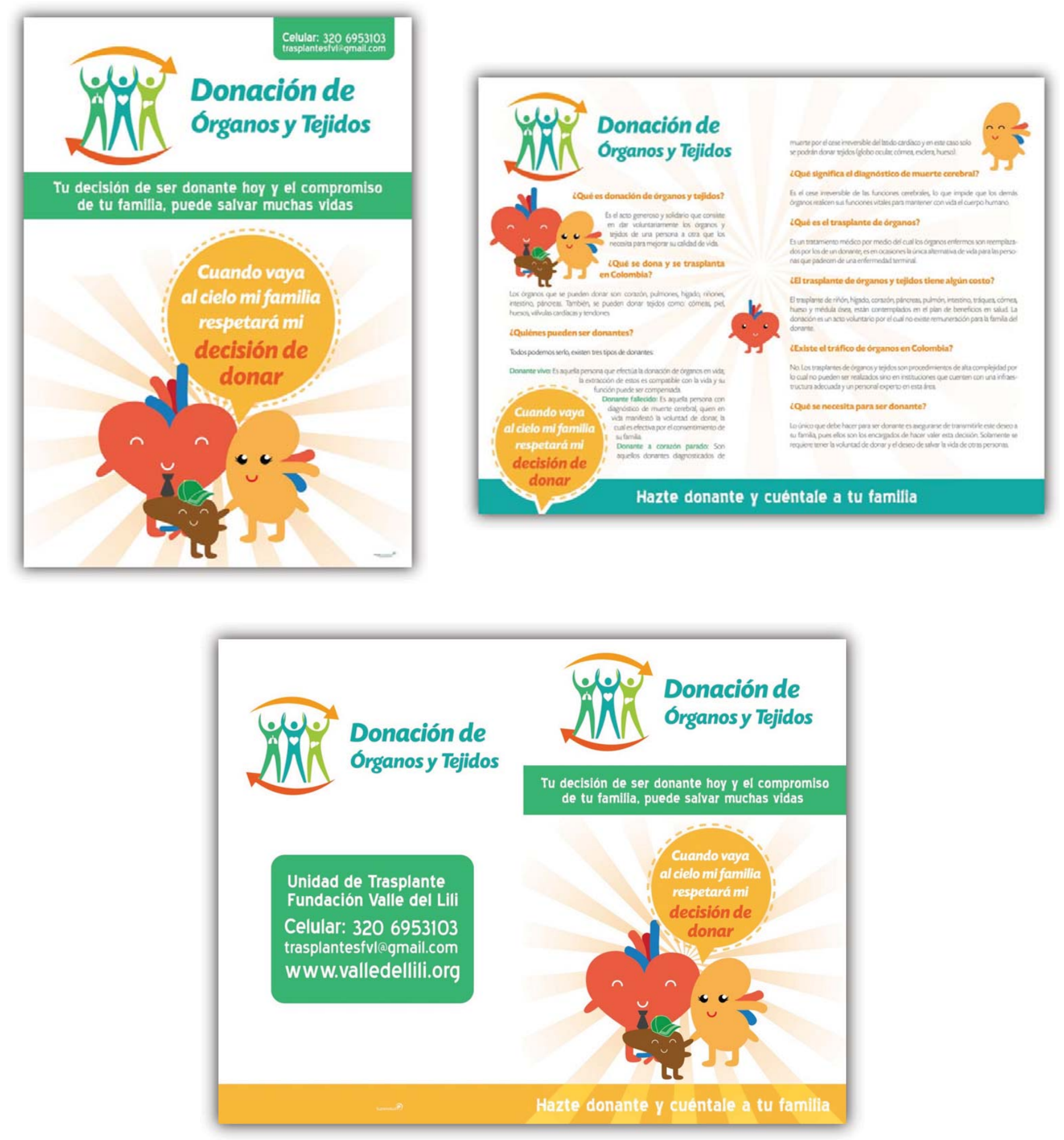

AnExo 2. Folletos informativos campaña "Hospital donante"

Creado y realizado por el Departamento de Comunicaciones, Fundación Valle del Lili, Cali, Colombia 